\title{
Projection optics design for tilted projection of fringe patterns
}

\author{
Yuan Shu \\ Ronald Chung \\ The Chinese University of Hong Kong \\ Department of Mechanical and Automation \\ Engineering \\ Hong Kong, China \\ E-mail: rchung@cuhk.edu.hk
}

\section{Zheng Tan}

Xi'an Jiatong University

School of Electronic and Information Engineering

Shannxi, China

\author{
Jun Cheng* \\ The Chinese University of Hong Kong \\ Department of Mechanical and Automation \\ Engineering \\ Hong Kong, China
}

Edmund Y. Lam, MEMBER SPIE

The University of Hong Kong

Department of Electrical and Electronic

Engineering

Hong Kong, China

Kenneth S. M. Fung

Fan Wang

ASM Assembly Automation Limited

Kwai Chung, N.T.

Hong Kong, China

\begin{abstract}
A challenge in the semiconductor industry is 3-D inspection of the miniaturized solder bumps grown on wafers for direct die-to-die bonding. An inspection mechanism proposed earlier requires the projection of a binary fringe grating to the inspected surface from an inclined angle. For high speed and accuracy of the mechanism, the projection optics has to meet these requirements: (1) it allows a tilt angle between the inspected surface and the projector's optical axis; (2) it has a high bandwidth to let high-spatial-frequency harmonics contained in the binary grating pass through the lens and be projected onto the inspected surface properly; (3) it has a high modulation transfer function; (4) it has a large field of view; and (5) it has an adequate depth of field that matches the depth range of the inspected surface. In this paper, we describe a projection optics design, consisting of a fringe grating and several pieces of spherical lens, that addresses the requirements. To reduce the lens aberrations, the grating is laid out with an angle chosen specifically to make the grating, the lens, and the average plane of the inspected surface intersect in the same line. Performance analysis and tolerance analysis are shown to demonstrate the feasibility of the design. (C) 2008 Society of Photo-Optical Instrumentation Engineers. [DOI: 10.1117/1.2931457]
\end{abstract}

Subject terms: machine vision; lens design; 3-D from structured light; modulation transfer function; depth of field; field of view.

Paper 070738R received Sep. 24, 2007; revised manuscript received Feb. 10 , 2008; accepted for publication Mar. 9, 2008; published online May 30, 2008; corrected Jun. 3, 2008.

\section{Introduction}

With the semiconductor industry advancing rapidly toward smaller, lighter, faster, and cheaper products, area array packages, including ball grid arrays, chip-scale packages, flip-chip packaging, wafer bumping, and wafer-level packaging (WLP), are quickly becoming the focus of IC packaging technology. They could help improve device performance and manufacturability, and ultimately reduce cost.

However, the shrunk dimension of the devices also leads to more stringent requirement on process control and quality assurance. A particular challenge is the development of vision techniques for inspecting the 3-D surface of wafer bumps accurately and efficiently. There have been a few noncontact optical shape measurement methods proposed in the literature: laser triangulation, ${ }^{1-3}$ confocal microscopy, ${ }^{4,5}$ sinusoidal pattern projection, ${ }^{6}$ and moiré interferometry. ${ }^{7,8}$ However, these methods suffer from either low speed or high sensitivity to noise. In an earlier work we proposed a new mechanism for reconstructing wa-

\footnotetext{
* Current affiliation: Shenzhen Institute of Advanced Integration Technology, Chinese Academy of Sciences/The Chinese University of Hong Kong.
}

0091-3286/2008/\$25.00 @ 2008 SPIE fer bump surfaces in $3-\mathrm{D} .{ }^{9,10}$ As shown in Fig. 1, the grating is projected onto the inspected surface at an incident angle of $30 \mathrm{deg}$. The mechanism is based on projecting a binary pattern to the surface and capturing image of the illuminated scene. By shifting the binary pattern in

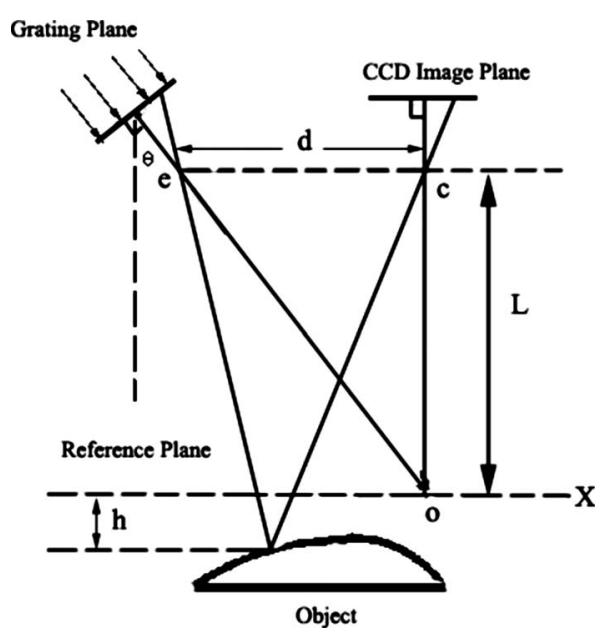

Fig. 1 3-D wafer measurement system. 

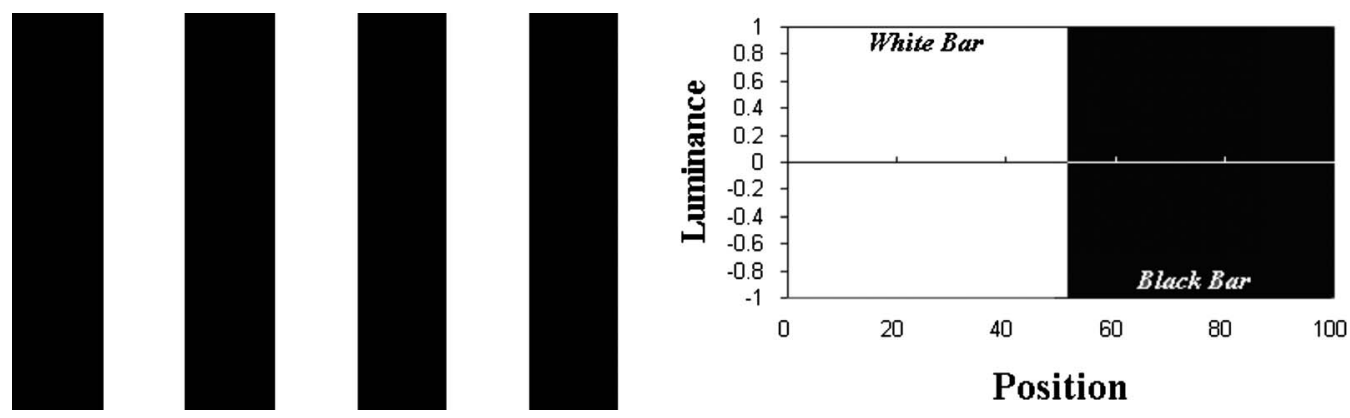

Fig. 2 Luminance of a binary grating.

space and every time taking a separate image of the illuminated surface, each position on the illuminated surface will be assigned a binary code in the sequence of images taken. With a suitable design of the binary pattern, the binary code can be made unique for each bump surface position. With such binary codes, correspondences between image positions and positions of the projected pattern can be readily established. 3-D information about the bump surface can then be obtained over these coded points via triangulation.

The design of the projection lens, which determines the quality of the projected fringes on the inspected surface, is important for the proposed inspection system. To reconstruct 3-D surfaces with high speed and accuracy, there are the following requirements for the projection lens system: (1) it allows a tilt angle of about $60 \mathrm{deg}$ between the inspected surface and the projector's optical axis; (2) it has a large bandwidth to let high-spatial-frequency harmonics contained in the binary grating pass through and be projected onto the inspected surface properly; (3) it has a large modulation transfer function (MTF); (4) it has a large field of view (FOV) of about $10 \times 10 \mathrm{~mm}$ over the inspected surface, to speed up the reconstruction process; (5) it has a large depth of field (DOF) that corresponds to the height of the inspected surface. The above requirements lead to great challenges in the projection lens design. This paper addresses the design of such a projection system.

The organization of the paper is as follows. Section 2 outlines the design issues, including the MTF requirement of the designed lens, the key factors for correcting lens aberration, and the methodology of improving the depth of field of the lens. Two designed lens systems that meet the requirements are described in Sec. 3. Concluding remarks are presented in Sec. 4.

\section{Design Issues}

The grating pattern projection system is to project a pattern onto the microsurface in such a way that the pattern, on reflection from the surface and imaging by the camera, will retain the fine details to a specified level. The main concerns in the design of the system are the defocus (modulation reduction) and image distortion (pattern variation) in the propagation of the light. The distortion will cause an offset of both the grating position and the pitch variation, which generally affect the 3-D (height) measurement; also, the modulation reduction will reduce the SNR and in turn the accuracy of the projected pattern edge detection.

\subsection{Image Modulation Requirement of the Projection System}

A binary pattern (stripe grating) is a repeating sequence of light and dark bars, with one pair of adjacent light and dark bars making up each cycle. These cycles repeat in a grating. These square gratings in the captured image data are supposed to be reducible to a set of sharp edges like the bars shown in Fig. 2.

A binary pattern $f(x)$ of period $2 L$ can be expressed by a Fourier series:

$f(x)=\frac{4}{\pi} \sum_{n=1,3,5, \ldots}^{\infty} \frac{1}{n} \sin \left(\frac{n \pi x}{L}\right)$.

It consists of more high-frequency components than a sinusoidal grating, and the amplitudes of the components get smaller as the frequencies go up. When the binary pattern is projected onto the inspected surface through a set of projection lenses, the high-order harmonics of the pattern will be diminished because of the lenses' limited bandwidth. Therefore, crisp fringes as shown in Fig. 2, when projected onto the inspected surface, will not remain perfectly black or white, but will appear as gray-level fringes. In other words, the boundaries of the binary stripes will appear blurred in the captured image data. When a binary grating with spatial frequency $\nu$ is projected on the reference plane

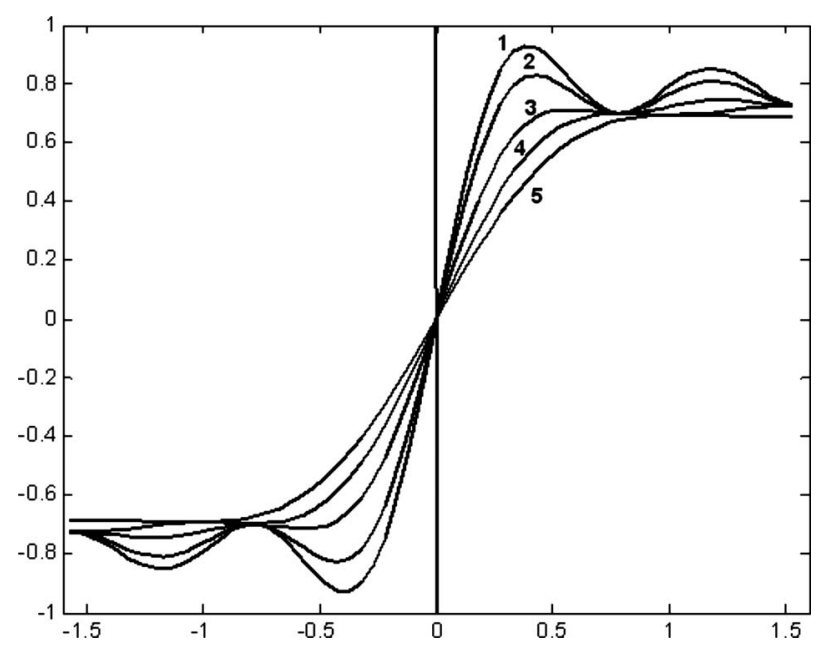

Fig. 3 Intensity distribution of edges with different MTF values. 


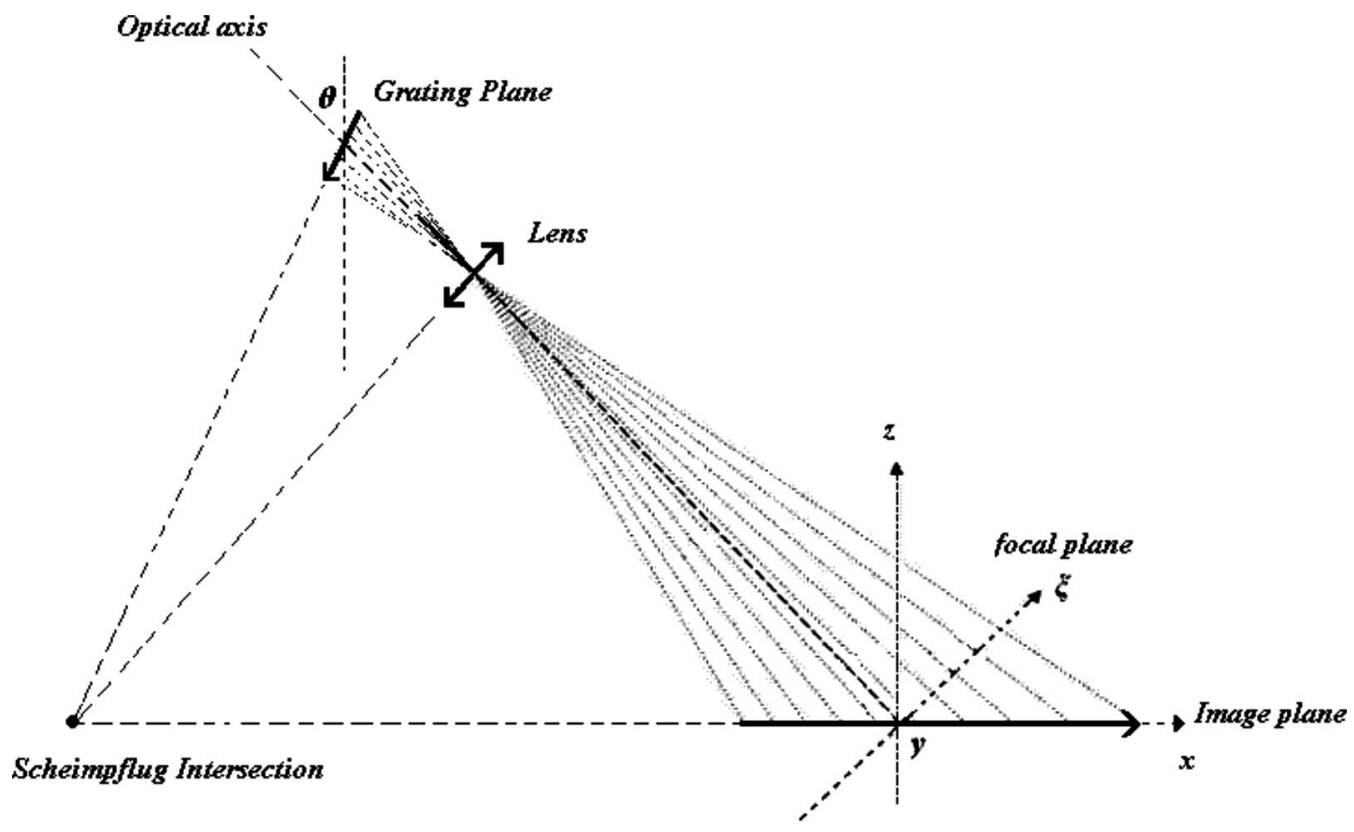

Fig. 4 Optical axis inclination setup of the grating projection unit.

through the projection lens, the modulation transfer function (MTF) of the projection lens is defined as

$\mathrm{MTF}=\frac{M_{i}(\nu)}{M_{o}(\nu)}$

where $M_{i}$ is the modulation of the projected fringes and $M_{o}$ is the modulation of the grating. The MTF is a function of the spatial frequency $\nu$, which decreases rapidly with increasing $\nu$.

The sharper is an edge, the higher frequency components it contains. Figure 3 illustrates a set of intensity profiles around the edge region, varying from smooth to sharp. The smooth edges do not contain enough high-frequency harmonics to present a rapid transition. Only with more higher-frequency harmonics retained could sharper edges be obtained. To sharpen the edge between black and white stripes in the projection, more harmonics of the proper frequency and amplitude should pass through the projection lens. ${ }^{11,}$

Experiments show that if in the image data we are to capture fringes with sharp enough edges on the inspected surface, we need to design a projection lens with a high MTF value for the fifth harmonic of the grating's spatial frequency. As our system requires a binary grating with 10 line pairs per millimeter $(1 \mathrm{p} / \mathrm{mm})$, we need to design a lens with good MTF performance at $50-1 \mathrm{p} / \mathrm{mm}$ spatial frequency.

\subsection{Aberration Corrections of the Cross-Axis Optics}

Various kinds of aberrations occur when light passes through the portion of the lens that is distant from the lens center. They include spherical aberration, coma aberration, chromatic aberration, and so on. ${ }^{13}$ Symmetric doublets can reduce the spherical aberration greatly. More complete coma aberration correction can be achieved by using a combination of lenses that are symmetric about a central stop, such as double Gauss lenses. One way to minimize the chromatic aberration is to use glasses of different dispersions in a doublet or in other combinations. In this work, we use a combination of different lenses to compensate the lens aberration.

Cross-axis layout of the CCD camera and the grating projector require that the image plane (i.e, the reference plane shown in Fig. 4, which is the average plane of the inspected surface) be not perpendicular but tilted with respect to the optical axis of the projector. In other words, the binary grating is projected onto the inspected surface at an oblique angle $\theta$. Large $\theta$ can improve the 3 -D reconstruction precision of the inspected wafer. However, it will greatly increase the lens aberration and reduce the MTF.

The first problem arising from this optical layout is that the lateral magnifications for the upper and lower edges of the image plane are different. This so-called keystone distortion is caused by the fringe pattern projection direction not being perpendicular to the image plane ${ }^{14,15}$ of the camera. The keystone distortion is not symmetric with respect to the optical axis; thus we cannot use the conventional

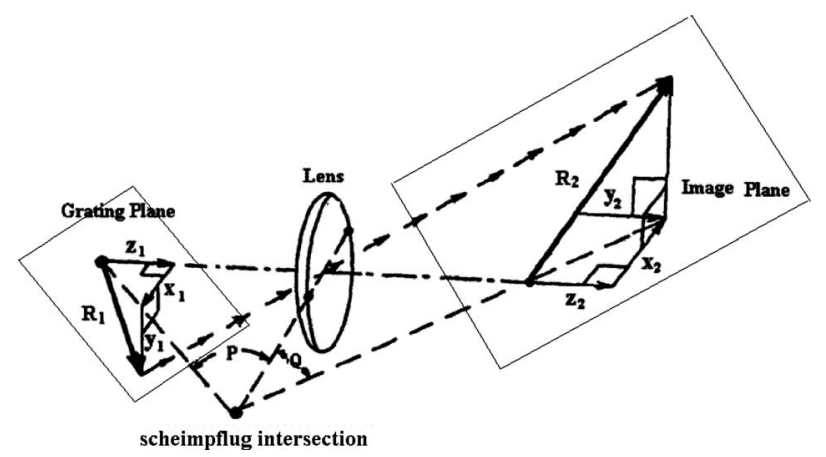

Fig. 5 Scheimpflug optics layout of the tilted grating and image plane. 


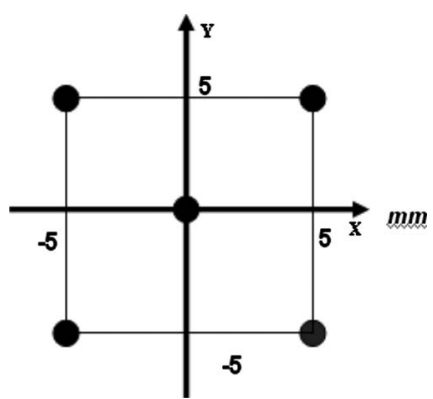

Fig. 6 Symmetrical field of view in the $10 \times 10-\mathrm{mm}$ image plane.

spherical lens combination for correcting it at all positions in the image plane. The second problem is that the focusing acuity of the image changes linearly from the lower to the upper edge of the image plane. The image can at any particular setting be in sharp focus only over a horizontal strip of it. The solution in our design is to use the Scheimpflug optical system to eliminate both the keystone distortion and the linear defocusing effect. It differs from the conventional techniques in that the object plane, lens plane, and image plane are not parallel to each other, but intersect in a common straight line. Furthermore, the lines where the grating and image planes intersect with the corresponding principal planes must have the same height, as shown in Fig. 5. We can see that the required tilting of the grating plane to eliminate the keystone distortion is in the opposite sense to that required for focusing the image over the entire image plane. ${ }^{15}$ The chief advantage of the Scheimpflug geometry is that a large depth of focus can be achieved. ${ }^{16}$

Let $R_{1}$ be an arbitrary vector in the grating plane inclined at angle $P$ to the axis of the input lens, and $R_{2}$ be the projected image of $R_{1}$ in the image plane from an inclination angle $Q$. Let $m$ be the magnification of the projection lens. Then $R_{1}$ can be decomposed into three orthogonal components $x_{1}, y_{1}$, and $z_{1}$ (shown in Fig. 5). Since the inclination of the grating plane is a constant angle $P$, the following relationship will always be satisfied:

$\tan P=z_{1} / x_{1}$,

$\tan Q=z_{2} / x_{2}$.

The components of the image $R_{2}$ of $R_{1}$ will be similarly designed so that $x_{2}, y_{2}, z_{2}$ are related to the grating components by

$x_{2}=-m x_{1}$,

$y_{2}=-m y_{1}$,

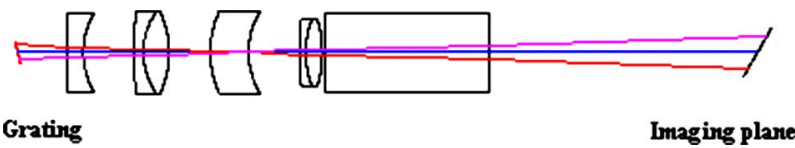

Fig. 7 3-D layout model of the projection lens.
Table 1 Specifications of the designed lens system with larger DOF.

\begin{tabular}{|c|c|c|c|}
\hline $\begin{array}{l}\text { Surface } \\
\text { no. }\end{array}$ & $\begin{array}{l}\text { Radius of curvature } \\
\qquad(\mathrm{mm})\end{array}$ & $\begin{array}{l}\text { Thickness } \\
(\mathrm{mm})\end{array}$ & Material \\
\hline Object & $\infty$ & 14.00 & Air \\
\hline 1 & $\infty$ & 3.00 & BK7 \\
\hline 2 & 24.53 & 14.15 & Air \\
\hline 3 & 82.92 & 3.00 & SF5 \\
\hline 4 & 21.38 & 7.89 & SK11 \\
\hline 5 & -28.75 & 7.60 & Air \\
\hline 6 & 26.68 & 11.49 & SF5 \\
\hline 7 & 21.84 & 20.09 & Air \\
\hline 8 & 83.79 & 2.00 & SF5 \\
\hline 9 & 28.12 & 5.00 & BK7 \\
\hline 10 & -35.92 & 6.89 & Air \\
\hline 11 & $\infty$ & 100.00 & BK7 \\
\hline 12 & $\infty$ & 63.39 & Air \\
\hline Image & $\infty$ & & \\
\hline
\end{tabular}

$z_{2}=m^{2} z_{1}$

where $m^{2}$ is the familiar longitudinal magnification.

The relation between the tilt angle $P$ of the grating pattern plane and the tilt angle $Q$ of the image plane can be expressed as

$\tan Q=-m \tan P$.

Using the method of tilting the grating plane, the offaxis keystone distortion can be corrected properly.

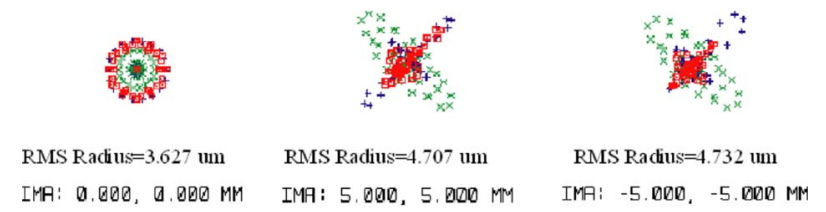

(a) Designed projection lens with small DOF
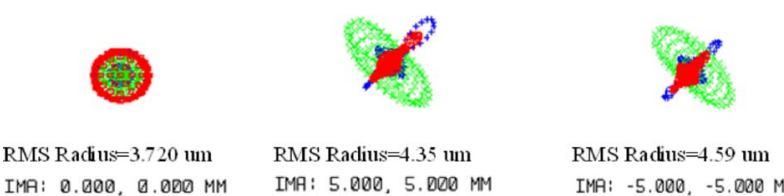

(b) Designed projection lens with large DOF

Fig. 8 Spot diagrams of three fields of view of the two projection lenses. 
Shu et al.: Projection optics design for tilted projection...

Table 2 Parameters of the two designed lens systems.

\begin{tabular}{lccccccc}
\hline \hline DOF & $\begin{array}{c}\text { Surface } \\
\text { no. }\end{array}$ & $\begin{array}{c}\text { Distance } \\
\text { OI } \\
(\mathrm{mm})\end{array}$ & $\begin{array}{c}\text { Focal } \\
\text { length } \\
(\mathrm{mm})\end{array}$ & NA & $\begin{array}{c}\text { Aperature } \\
(\mathrm{mm})\end{array}$ & Magnification & $\begin{array}{r}\text { MTF } \\
@ 50 \\
\text { Ip/mm }\end{array}$ \\
\hline Small & 14 & 210.48 & 39.72 & 0.1 & 9.05 & -2.134 & 0.52 \\
Large & 14 & 244.52 & 40.88 & 0.1 & 8.00 & -2.62 & 0.44 \\
\hline \hline
\end{tabular}

\subsection{Improving the Depth of Field for Bumped Ball Imaging}

When a lens focuses on an object at a distance, only the parts of the object that are exactly at that depth are in focus. Other parts are out of focus. The zone of acceptable sharpness is referred to as the depth of field (DOF). Since the average height of the bump is from 80 to $400 \mu \mathrm{m}$, the optics should have a DOF of about $400 \mu \mathrm{m}$. Increasing the
DOF of the projection lens increases the sharpness of the deformed pattern image. The DOF can be calculated as

$\Delta L_{1}=\frac{F \delta L^{2}}{f^{2}+F \delta L}$,

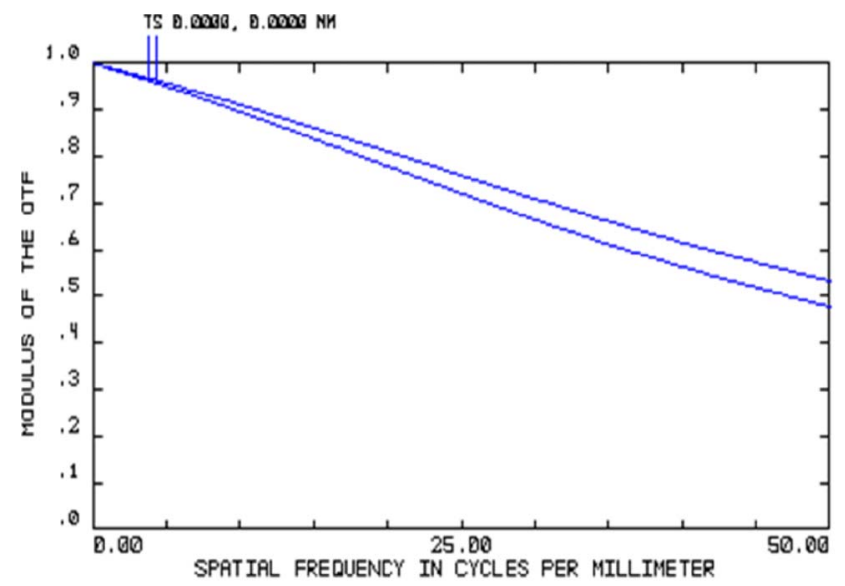

(a)

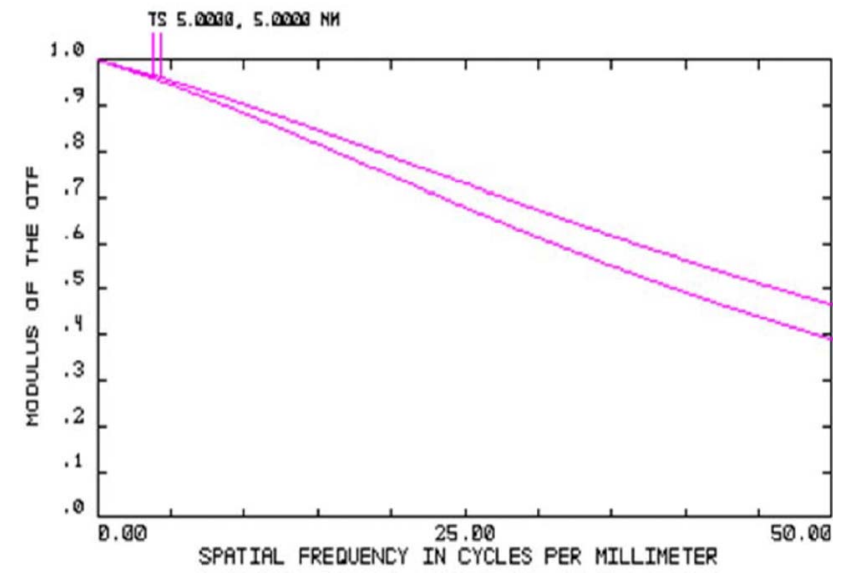

(b)

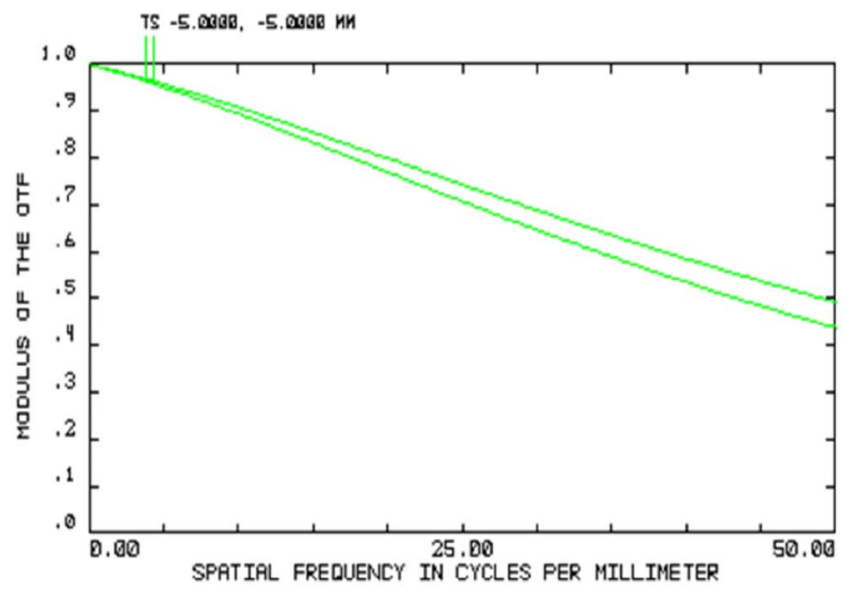

(c)

Fig. 9 MTF of the small-DOF design. 
$\Delta L_{2}=\frac{F \delta L}{f^{2}-F \delta L}$,

$\Delta L=\Delta L_{1}+\Delta L_{2}=\frac{2 f^{2} F \delta L^{2}}{f^{4}-F \delta^{2} L^{2}}$,

where $\delta$ is the diameter of the acceptable circle of confusion, $f$ is the focal length of the lens, $F$ is the aperture (or $F$ stop), $L$ is the focused subject distance (this is what is set on the lens focus scale), $\Delta L_{1}$ and $\Delta L_{2}$ are the near and far distance values of the DOF, respectively, and $\Delta L$ is the whole DOF of the optics. From Eq. (6), we know that the DOF can be increased by reducing the lens's aperture.

\section{Projection System Design and Performance Analysis}

The projection lens is required to properly project the binary grating of spatial frequency $10 \mathrm{lp} / \mathrm{mm}$ to the inspected surface with FOV of $10 \times 10 \mathrm{~mm}$. In order to capture the deformed fringes with sharp edges, a high modulation at the fifth harmonic of the base frequency is needed. The ZEMAX software ${ }^{17}$ is used in this optics design. The comprehensive software tool for optics design integrates all the features required to conceptualize, design, optimize, analyze, tolerance-insert, and document virtually any optical system.

\subsection{Designed Projection System}

Consider a FOV $10 \times 10 \mathrm{~mm}$. Five symmetrical positions, as shown in Fig. 6, are used for performance analysis. The purpose of the design is to optimize all parameters of the optics: radii, thickness, glasses, conics, aspheric coefficients, and so on. The layout of the designed projection optics, whose aperture is $9.05 \mathrm{~mm}$, is shown in Fig. 7 .

In order to improve the DOF of the projection lens, the aperture of the first designed optics was reduced to $8 \mathrm{~mm}$. The modified design with a larger DOF is shown in Fig. 7. The components' parameters for the modified design are shown in Table 1.

The parameters of the two designed systems are shown in Table 2, where the distance $O I$ is the total length from the grating to the image of the lens. From Table 2, we can see that as the aperture is reduced from 9.05 to $8.00 \mathrm{~mm}$, the MTF is also reduced a little, from 0.52 to 0.44 .

As for the designed system with small DOF, the tilt angle of the image plane is $Q=30 \mathrm{deg}$, the tilt angle of the grating is $P_{1}=15.04 \mathrm{deg}$, and the optical paraxial magnification $m_{1}$ is -2.134 . From Eq. (5), it can be determined that the relation between the tilted image and grating plane is $\tan Q / \tan P_{1} \approx-m_{1}$.

For the designed system with the larger DOF, the tilt angle of the image plane is also $Q=30 \mathrm{deg}$, the tilt angle of the grating pattern is $P_{2}=12.35 \mathrm{deg}$, and the optical paraxial magnification $m_{2}$ is -2.62 . The relation between the tilted image and grating plane of this modified design is $\tan Q / \tan P_{2} \approx-m_{2}$.

\subsection{Performance Analysis}

An ideal lens will focus an input parallel beam to a point (focal point), which should be infinitesimally tiny. However, because of lens aberrations and diffraction, the focal

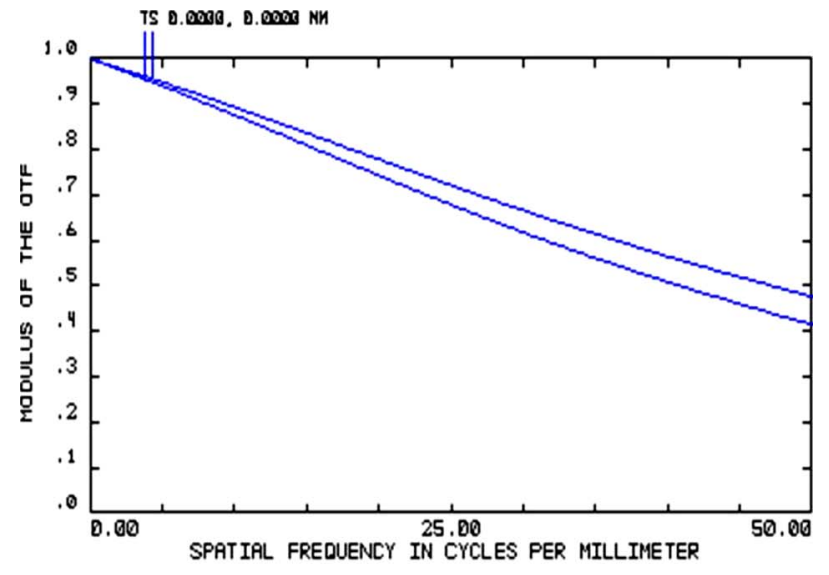

(a)

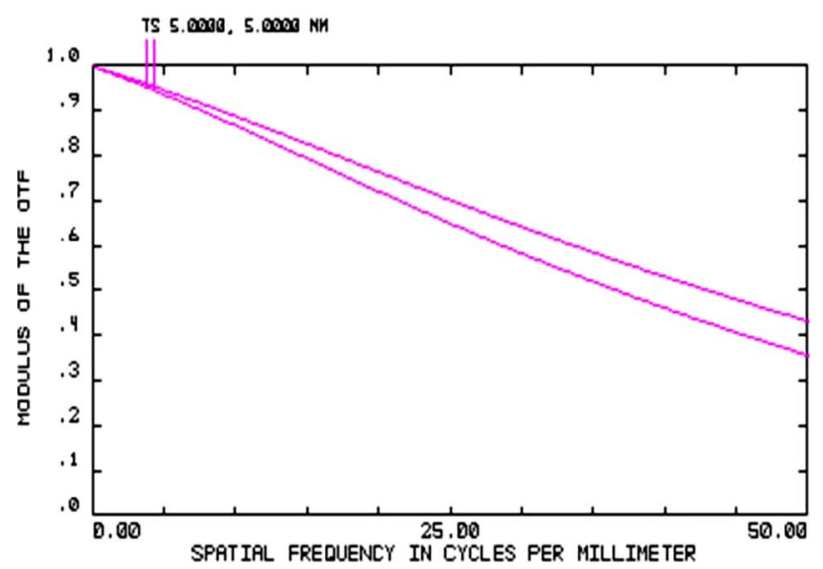

(b)

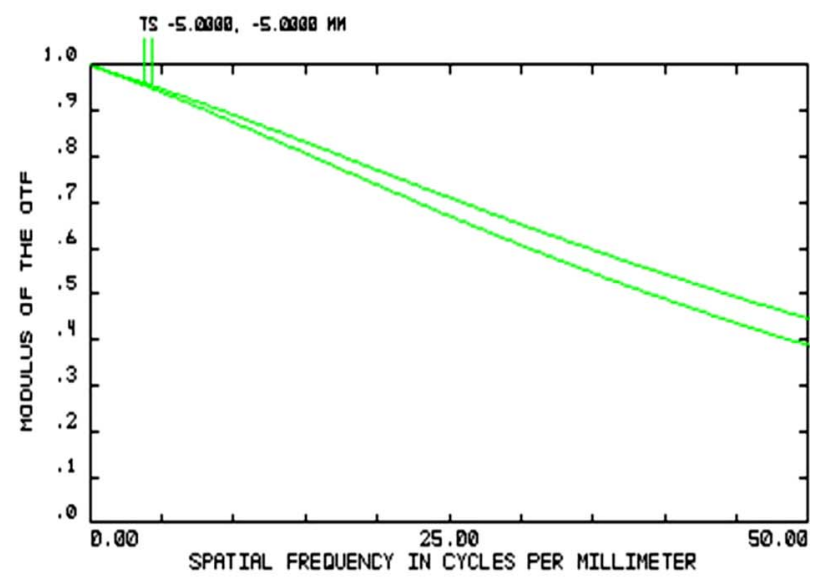

(c)

Fig. 10 MTF of the large-DOF design.

spot of a real lens has a finite size. All singlet lenses have a significant amount of aberration. Figure 8 shows a so-called spot diagram in the case that the grating plane and the image plane are placed at right angles to the optical axis of projection. In general, elimination of the keystone distortion can have the effect that the average image spot size at the edge of the field is increased.

To describe the lens's ability to transfer contrast at a 


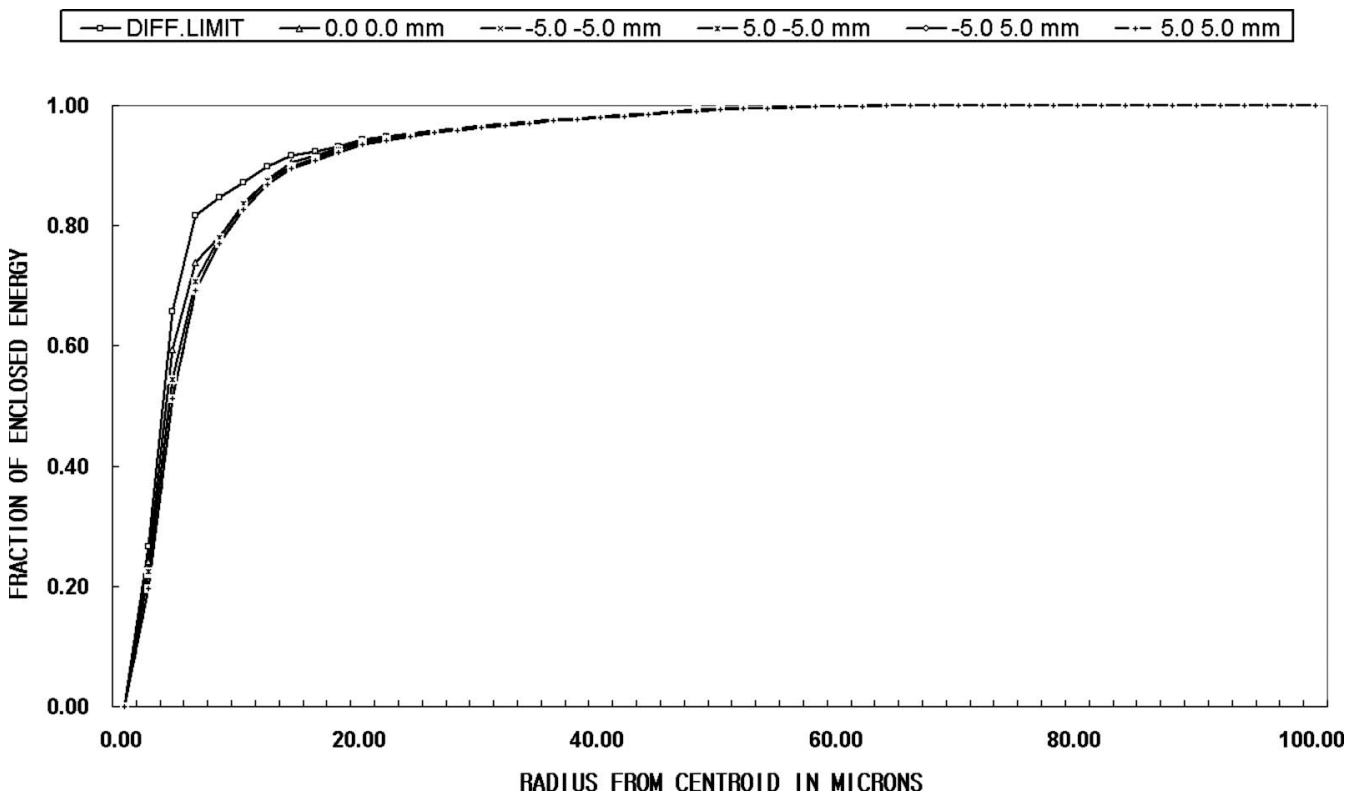

Fig. 11 Encircled-energy curves of the large-DOF design.

particular resolution level from the grating to the image, the MTF curves at three image positions $(0,0),(5,5)$, and $(-5,-5)$ are shown in Fig. 9 and Fig. 10. In each graph, there are two curves, one for the tangential MTF and the other for the sagittal MTF. The reason for the difference between the two plots is that the point spread function becomes asymmetric off axis, which means the resolution in the two directions is also different.

A graph of the encircled fraction of the energy plotted against the radius of the aperture is called the radial energy distribution curve. The curve (shown in Fig. 11) indicates the fraction $E$ of the total energy in an image pattern that falls in a circle of radius $R$.

If the acceptable sharpness in imaging target object is specified by a MTF at $50 \mathrm{lp} / \mathrm{mm}$ of 0.3 , the DOF can be improved from 380 to $470 \mu \mathrm{m}$ when the aperture is reduced from 9 to $8 \mathrm{~mm}$, as shown in Fig. 12. Such a design

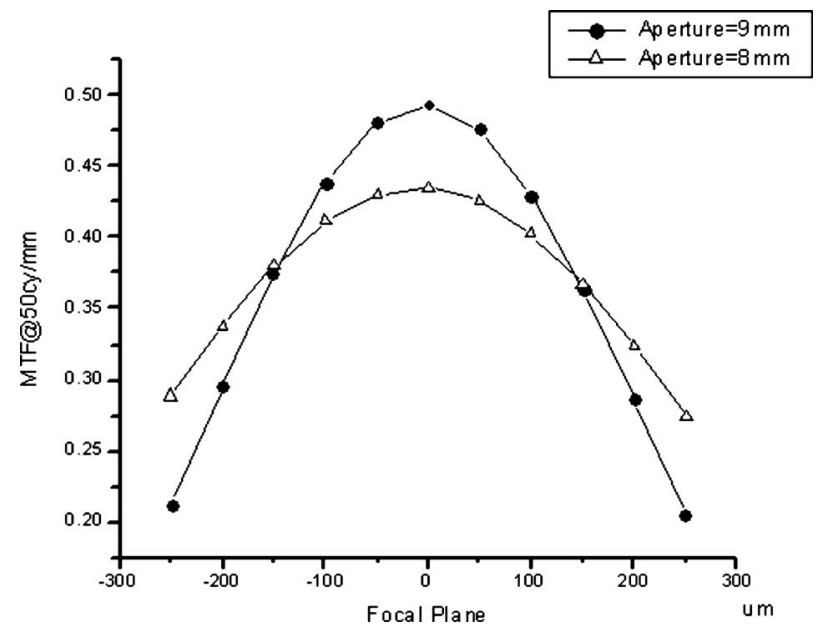

Fig. 12 Depths of field of the two projection lenses at $(0,0)$. with higher DOF can cover the average height of the inspected bumps, which is from 80 to $400 \mu \mathrm{m}$. The MTF parameters are reduced only a little, from 0.48 to 0.43 at position $(0,0)$ in the image plane.

\subsection{Tolerance Analysis}

Through the MTF curve shows what a lens is capable of theoretically under the optical prescription, it does not indicate the actual performance of the lens after manufacturing. Manufacturing always introduces some performance loss in the design, due to tolerances. For this reason, tolerance testing enables characterization of the actual performance of both the designed lens and the manufactured lens. Table 3 and Table 4 give the tolerance data and the simulated MTF parameters after considering the tolerance of the manufacturing, as well as the performance requirements of the optical system.

Note that theoretically with the use of more component lenses the performance of a lens system can be further im-

Table 3 Typical optical fabrication tolerance. ${ }^{13}$

\begin{tabular}{|c|c|c|c|c|c|}
\hline Grade & $\begin{array}{l}\text { Diameter } \\
(\mathrm{mm})\end{array}$ & $\begin{array}{c}\text { Thickness } \\
\text { (mm) }\end{array}$ & Radius & $\begin{array}{c}\text { Linear } \\
\text { dimension } \\
(\mathrm{mm})\end{array}$ & Angles \\
\hline Low cost & \pm 0.2 & \pm 0.5 & Gauge & \pm 0.5 & Degrees \\
\hline Commercial & \pm 0.07 & \pm 0.25 & $10 \mathrm{fr}$. & \pm 0.25 & $\begin{array}{c} \pm 15 \\
\text { arcmin }\end{array}$ \\
\hline Precision & \pm 0.02 & \pm 0.1 & $5 \mathrm{fr}$. & \pm 0.1 & $\begin{array}{l} \pm(5 t o 10) \\
\text { arcmin }\end{array}$ \\
\hline $\begin{array}{l}\text { Extra } \\
\text { precise }\end{array}$ & \pm 0.01 & \pm 0.05 & $\begin{array}{l}\text { As } \\
\text { reqd. }\end{array}$ & $\begin{array}{l}\text { As } \\
\text { reqd. }\end{array}$ & Arcseconds \\
\hline
\end{tabular}




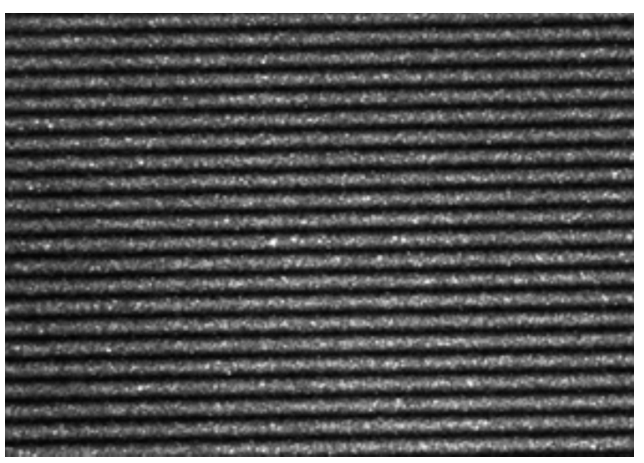

(a) Image of reference plane under pattern projection

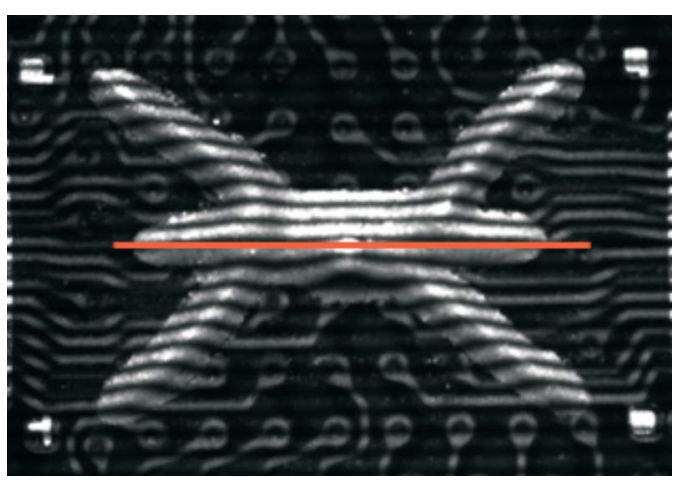

(b) Image of target object under pattern projection

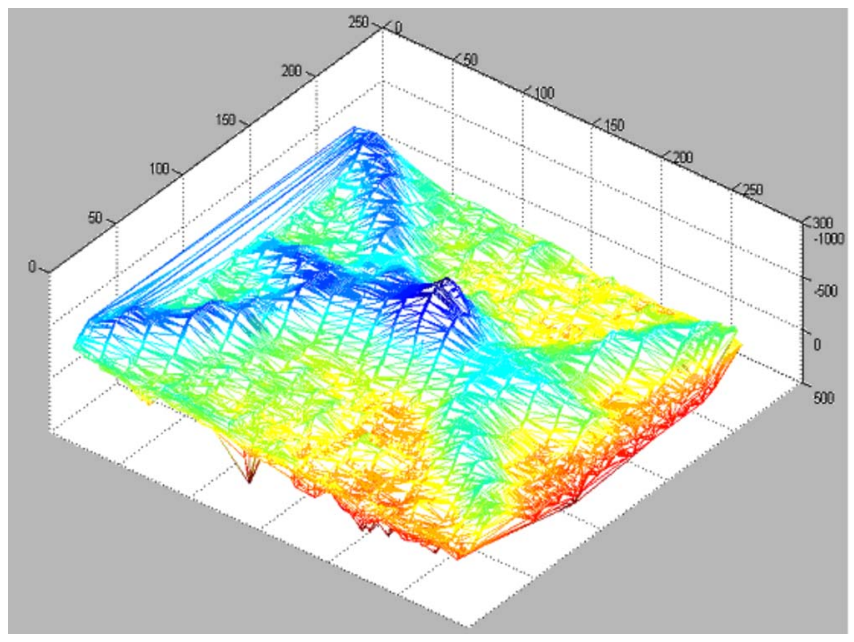

(c) Reconstructed height profile of object

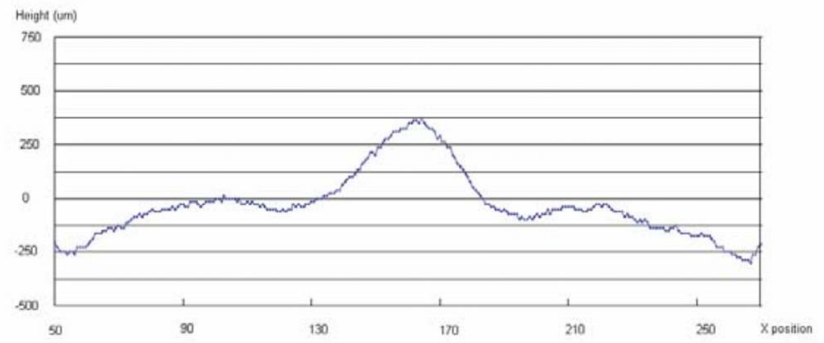

(d) 2-D plot of a cross section of the reconstructed

height profile from image position $(50,108)$ to $(280,108)$

on (b).

Fig. 13 3-D reconstruction of a small solder surface with the use of the grating projection system. 
Table 4 Tolerance tests (Monte Carlo, 20 repetitions) on the two designed lenses.

\begin{tabular}{lccc}
\hline \hline & \multicolumn{3}{c}{ MTF at $50 \mathrm{lp} / \mathrm{mm}$} \\
\cline { 2 - 4 } DOF & $90 \%$ & $50 \%$ & $10 \%$ \\
\hline Small & $>0.36$ & $>0.43$ & $>0.48$ \\
Large & $>0.33$ & $>0.39$ & $>0.42$ \\
\hline \hline
\end{tabular}

proved. However, the use of more lenses could also bring about larger tolerances in the manufacturing process and affect the actual optical performance. The manufacturing cost will also increase.

\subsection{Real Image Experiments}

Experiments have been conducted to inspect the microsolder epoxy on an IC packaging device surface, for which the proposed projection system is a key component of the reconstruction system. In the result presented in Fig. 13, the height distribution of a very small solder surface (the whole object surface was no bigger than $12 \mathrm{~mm} \times 12 \mathrm{~mm}$ $\times 600 \mu \mathrm{m})$ was inspected to show the 3 -D profile measurement capability of the system for small-form parts. The reconstruction system, detailed in Refs. 9 and 10, uses the proposed projection system to project the fringe grating first to a reference plane and then to the target surface. The image data taken separately for the two surfaces are then compared through an algorithm, and 3-D measurements of the target surface can be obtained with reference to the reference plane. Figure 13(a) shows an image of the reference plane under the pattern projection. It was part of a calibration step. Figure 13(b) shows the image of the target object under pattern projection. Figure 13(c) shows the reconstructed height distribution of the object. Figure 13(d) shows a 2-D plot indicating a cross section of the reconstructed solder epoxy profile height from image position $(50,108)$ to $(280,108)$ (the red line) in Fig. 13(b).

Thorough evaluation experiments against ground truth have been conducted. The ground truth was measured by a machine of high precision and accuracy-the ZYGO machine - and the solder epoxy profile was detected using a phase-shifting interferometer with $0.1-\mu \mathrm{m}$ height resolution. The comparison shows that in the direction of the height measurement a mean squared error of no more than $9.43 \mu \mathrm{m}$ was achieved by the 3-D reconstruction system, and that confirms the validity of the proposed grating projection system design in achieving quality $3 \mathrm{D}$ reconstruction.

\section{Conclusion}

To realize an optical system for semiconductor surface inspection, a design of a projection lens system with the following requirements is needed: (1) it allows a tilt between the inspected surface and the projector's optical axis; (2) it has large bandwidth; (3) it has large MTF; (4) it has large FOV; and (5) it has large DOF. We have described how the design challenge can be met using a combination of lenses and a method of correcting keystone aberration. The designed projection lens system can project a binary grating onto the inspected surface with sharp contrast between the light and dark fringes. Theoretical analysis demonstrates the feasibility of the design.

\section{Acknowledgment}

The work described in this paper was substantially supported by a grant from the Innovation and Technology Commission of Hong Kong Special Administrative Region, China, under an Innovation and Technology Fund with Project Code UIM/111.

\section{References}

1. H. Stern, "Laser based 3-d surface mapping for manufacturing diagnostics and reverse engineering," in Proc. IEEE Conf. on Aerospace and Electronics, pp. 1205-1212 (1992).

2. S. Wang, B. Zhuang, and W. Zhang, "New principle of optical displacement measurement based on light scattering from rough surface," in Three-Dimensional and Laser-Based Systems for Metrology and Inspection II, K. Harding and D. Svetkoff, Eds., Proc. SPIE 2909, 37-42 (1997).

3. C. Tay, S. Wang, C. Quan, B. Lee, and K. Chan, "Measurement of a micro-solderball height using a laser projection method," Opt. Commun. 234, 77-86 (2004).

4. M. Ishihara and H. Sasaki, "High-speed 3d shape measurement using a nonscanning multiple-beam confocal imaging system," in Laser Interferometry IX: Techniques and Analysis, M. Kujawinska, G. M. Brown, and M. Takeda, Eds., Proc. SPIE 3478, 68-75 (1998).

5. A. Rao, N. Ramesh, F. Wu, J. Mandeville, and P. Kerstens, "Algorithms for a fast confocal optical inspection system," in Proc. IEEE Workshop on Applications of Computer Vision, pp. 298-305 (1992).

6. V. Srinivasan, H. Liu, and M. Halioua, "Automated phase-measuring profilometry of 3-d diffuse objects," Appl. Opt. 23, 3105-3108 (1984).

7. C. J. Tay, S. H. Wang, and C. Quan, "Height measurement of microchip connecting pins by use of stereovision," Opt. Eng. 43, 963-970 (2004).

8. S. Kim, Y. Choi, and J. Oh, "Three-dimensional profile measurement of fine object by phase-shifting moiré interferometry," in ThreeDimensional and Laser-Based Systems for Metrology and Inspection II, K. Harding and D. Svetkoff, Eds., Proc. SPIE 2909, 28-36 (1997).

9. J. Cheng, R. Chung, E. Lam, K. Fung, F. Wang, and W. Leung, "Three-dimensional reconstruction of wafer solder bumps using binary pattern projection," in Machine Vision Applications in Industrial Inspection XIII, J. R. Price and F. Meriaudeau, Eds., Proc. SPIE 5679, 44-52 (2005).

10. J. Cheng, R. Chung, E. Lam, K. Fung, F. Wang, and W. Leung, "Structured-light based sensing using a single fixed fringe grating: fringe boundary detection and $3 \mathrm{~d}$ reconstruction," IEEE Trans. Electron. Packag. Manuf. 31(1), 19-31 (2008).

11. J. Goodman, Introduction to Fourier Optics, McGraw-Hill, New York (1996).

12. M. Born and B. Wolf, Principles of Optics, Pergamon Press, Oxford (1987).

13. J. S. Warren, Modern Optical Engineering: The Design of Optical Systems, McGraw-Hill (1990).

14. B. Li and L. Sezan, "Automatic keystone correction for smart projectors with embedded camera," in Proc. IEEE Int. Conf. on Image Processing, Vol. 4, pp. 2829-2832 (2004).

15. V. M. Durán-Ramírez and D. Malacara-Doblado, "Keystone aberration correction in overhead projectors," Appl. Opt. 43, 4123-4126 (2004).

16. A. K. Prasad and K. Jensen, "Scheimpflug stereocamera for particle image velocimetry in liquid flows," Appl. Opt. 34, 7092-7099 (1995).

17. ZEMAX Development Corp., http://zemax.com/zemax/ (2004). 


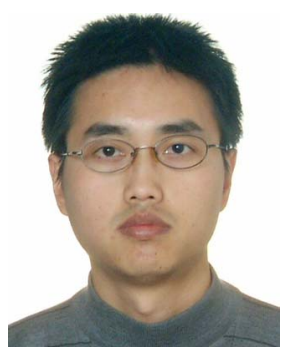

Yuan Shu received his $\mathrm{PhD}$ degree in communication and information systems from the Institute of Signal Processing of Xi'an Jiaotong University, China, in December 2005. Before that, he received his BS and MS degrees in applied physics and optics, both from Xi'an Jiaotong University, 1998 and 2001, respectively. He was a research assistant with the Computer Vision Laboratory (CVL), Department of Mechanical and Automation Engineering, the Chinese University of Hong Kong (CUHK), between November 2004 and April 2005. Now, he is a computer vision software engineer in a global semiconductor equipment company. His research interests are in 3-D reconstruction, high-precision pattern alignment, microdefect inspection, and machine vision.

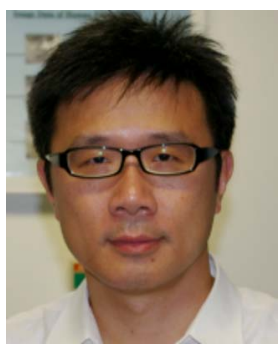

Ronald Chung received his BSEE from the University of Hong Kong, Hong Kong, and his $\mathrm{PhD}$ in computer engineering from University of Southern California, Los Angeles. $\mathrm{He}$ is currently with the Chinese University of Hong Kong, Hong Kong, as director of the Computer Vision Laboratory and professor in the Department of Mechanical and Automation Engineering. His research interests include computer vision and robotics. $\mathrm{He}$ is a senior member of the IEEE, a chartered engineer of the Engineering Council of the UK, and a member of Mensa. He was the chairman of the IEEE Hong Kong Section Joint Chapter on Robotics \& Automation Society and the Control Systems Society in the years 2001 to 2003.

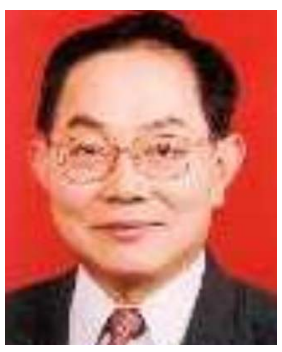

Zheng Tan received the BA degree from the Department of Electronic Engineering of Xi'an Jiaotong University in 1960. Since 1984, he has been with the Institute of Signal Processing of Xi'an Jiaotong University, China. Now he is a professor and a PhD student supervisor. His research interests are in stereo vision, digital entertainment, and virtual reality. He has published more than 60 papers in national and international journals and at international conferences.

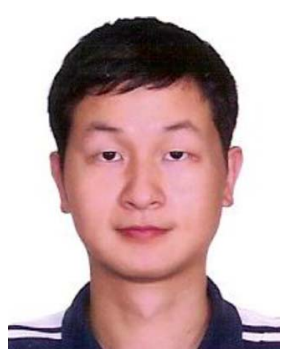

Jun Cheng received bachelor of engineering, bachelor of finance, and master of engineering degrees from the University of Science \& Technology of China in 1999 and 2002. His $\mathrm{PhD}$ degree was awarded at the Chinese University of Hong Kong in 2006. Currently he is with Shenzhen Institute of Advanced Integration Technology, Chinese Academy of Sciences/The Chinese University of Hong Kong, as a research associate fellow and director of the Laboratory for Semiconductor Equipment. His research interests include computer vision, robotics, machine intelligence, and control.

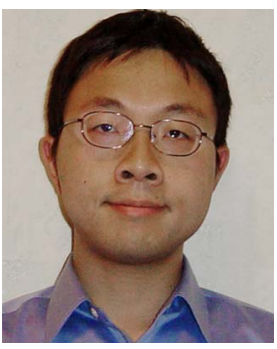

Edmund Y. Lam received the BS degree in 1995, the MS degree in 1996, and the PhD degree in 2000 , all in electrical engineering, from Stanford University. At Stanford, he was a member of the Information Systems Laboratory, conducting research for the Stanford Programmable Digital Camera project. His focus was on developing image restoration algorithms for digital photography. Outside Stanford, he also consulted for industry in the areas of digital camera systems design and algorithms development. Before returning to academia, he worked in the Reticle and Photomask Inspection Division (RAPID) of KLA-Tencor Corporation in San Jose as a senior engineer. His responsibility was to improve on the core die-to-die and die-to-database inspection algorithms, especially for phase-shift masks. Dr. Lam is currently an assistant professor in the Department of Electrical and Electronic Engineering at the University of Hong Kong. $\mathrm{He}$ is a member of the IEEE and SPIE.

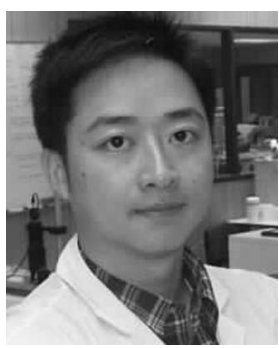

Kenneth S. M. Fung is the technical manager in the Research and Development Department at ASM Assembly Automation Limited. After receiving the $\mathrm{PhD}$ degree in 1999 from the Department of Electrical and Electronic Engineering at the University of Hong Kong, he joined ASM Assembly Automation Limited as a senior engineer. He developed a subpixel-accuracy, high-speed, and robust computer vision alignment algorithm that was applied in all ASM products and boosted the capability and vision technology level of the company's semiconductor packaging machines. Currently, he leads a team of research engineers responsible for the projects in machine vision inspection and algorithm development. He also provides technical supervision for a team of vision application engineers who are responsible for the projects in developing machine vision applications on ASM products. Kenneth's research interests are computer vision, pattern recognition, digital image processing, and artificial neural networks.

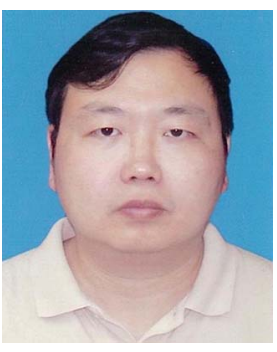

Fan Wang finished his undergraduate study in Tianjin University, China, in 1985, and obtained a master in physics degree from South China University of Technology, China, in 1988. He subsequently went back to Tianjin University, China, and obtained his PhD there in 1991. He has published on optical association memory and optical information processing. His recent interests include optical projector and 3-D display technologies. 